\title{
Comparison of inulin clearance with 2-h creatinine clearance in Japanese pediatric patients with renal disease: open-label phase 3 study of inulin
}

\author{
Osamu Uemura ${ }^{1} \cdot$ Kenji Ishikura $^{2} \cdot$ Koichi Kamei $^{3} \cdot$ Riku Hamada $^{4} \cdot$ Masaki Yamamoto $^{5} \cdot$ Yoshimitsu Gotoh $^{6}$. \\ Naoya Fujita ${ }^{7} \cdot$ Tomoyuki Sakai $^{8} \cdot$ Takafumi Sano $^{9} \cdot$ Masahiko Fushimi $^{9} \cdot$ Kazumoto lijima $^{10}$
}

Received: 7 June 2021 / Accepted: 6 September 2021 / Published online: 25 September 2021

(c) The Author(s) 2021

\begin{abstract}
Background There is no approved dosage and administration of inulin for children. Therefore, we measured inulin clearance (Cin) in pediatric patients with renal disease using the pediatric dosage and administration formulated by the Japanese Society for Pediatric Nephrology, and compared Cin with creatinine clearance (Ccr) measured at the same time. We examined to what degree Ccr overestimates Cin, using the clearance ratio (Ccr/Cin), and confirmed the safety of inulin in pediatric patients. Methods Pediatric renal disease patients aged 18 years or younger were enrolled. Inulin $(1.0 \mathrm{~g} / \mathrm{dL})$ was administered intravenously at a priming rate of $8 \mathrm{~mL} / \mathrm{kg} / \mathrm{hr}(\max 300 \mathrm{~mL} / \mathrm{hr}$ ) for $30 \mathrm{~min}$. Next, patients received inulin at a maintenance rate of $0.7 \times \mathrm{eGFR} \mathrm{mL} / \mathrm{min} / 1.73 \mathrm{~m}^{2} \times$ body surface area $(\max 100 \mathrm{~mL} / \mathrm{hr}$ ) for $120 \mathrm{~min}$. With the time the maintenance rate was initiated as a starting point, blood was collected at 30 and $90 \mathrm{~min}$, while urine was collected twice at 60-min intervals. The primary endpoint was the ratio of $\mathrm{Ccr}$ to $\mathrm{Cin}(\mathrm{Ccr} / \mathrm{Cin})$.

Results Inulin was administered to 60 pediatric patients with renal disease; 1 patient was discontinued and 59 completed. The primary endpoint, $\mathrm{Ccr} / \mathrm{Cin}$, was $1.78 \pm 0.52$ (mean \pm standard deviation). Regarding safety, five adverse events were observed in four patients (6.7\%); all were non-serious. No adverse reactions were observed in this study.

Conclusions The results in this study on the dosage and administration of inulin showed that inulin can safely and accurately determine GFR in pediatric patients with renal disease.

ClinicalTrials.gov identifier NCT03345316.
\end{abstract}

Keywords Inulin clearance $\cdot$ Creatinine clearance $\cdot$ Glomerular Filtration Rate $\cdot$ Chronic kidney disease $\cdot$ Children

Takafumi Sano

t-sano@fujiyakuhin.co.jp

1 Department of Pediatrics, Ichinomiya Medical Treatment \& Habilitation Center, 1679-2 Tomida-nagaresuji, Ichinomiya-shi, Aichi 494-0018, Japan

2 Department of Pediatrics, Kitasato University School of Medicine, 1-15-1 Kitazato, Minami-ku, Sagamihara-shi, Kanagawa 252-0374, Japan

3 Department of Nephrology and Rheumatology, National Center for Child Health and Development, 2-10-1 Okura, Setagaya-ku, Tokyo 157-8535, Japan

4 Department of Nephrology, Tokyo Metropolitan Children's Medical Center, 2-8-29 Musashidai, Fuchu-shi, Tokyo 183-8561, Japan

5 Department of Pediatrics, Seirei Hamamatsu General Hospital, 2-12-12 Sumiyoshi, Naka-ku, Hamamatsu-shi, Shizuoka 430-8558, Japan
6 Department of Pediatric Nephrology, Japanese Red Cross Nagoya Daini Hospital, 2-9 Myoken-cho, Showa-ku, Nagoya-shi, Aichi 466-8650, Japan

7 Department of Pediatric Nephrology, Aichi Children's Health and Medical Center, 7-426 Morioka-cho, Obu-shi, Aichi 474-8710, Japan

8 Department of Pediatrics, Shiga University of Medical Science, Tsukinowa, Seta, Otsu-shi, Shiga 520-2192, Japan

9 Development Department, Research Institute, Fuji Yakuhin Co., Ltd, 4-383, Sakuragi-cho, Omiya-ku, Saitama-shi, Saitama 330-9508, Japan

10 Department of Pediatrics, Kobe University Graduate School of Medicine, 7-5-2 Kusunoki-Cho, Chuo-ku, Kobe 650-0017, Japan 


\section{Introduction}

Inulin is uniformly distributed in extracellular fluid, filtered by the renal glomerulus, and then excreted in the urine without being secreted or reabsorbed by the renal tubules due to its physical and chemical properties [1]. In addition, because inulin does not bind to plasma proteins and circulates in the body without being metabolized, inulin clearance (Cin) is regarded as the gold standard for glomerular filtration rate (GFR) determination. However, since the method used to measure Cin is complicated and imposes a heavy burden on children, renal function in pediatric patients is usually evaluated using the estimated GFR (eGFR) calculated from creatinine (Cre), cystatin $\mathrm{C}$ (Cys-C) or beta 2-microglobulin (BMG), and creatinine clearance (Ccr). Serum Cre (sCre) is widely used as a renal function marker in clinical practice, but it is known that Ccr overestimates the actual GFR because Cre is excreted in the urine by secretion into renal tubules in addition to glomerular filtration [2]. Furthermore, since Cre is produced in skeletal muscle and sCre is proportional to muscle mass, eGFR calculated from sCre levels does not always accurately reflect the actual GFR in pediatric patients with significantly less muscle mass due to severe psychosomatic disorders, neuromuscular disease, and poor nutrition [3]. For such patients, renal function should be evaluated by eGFR calculated from serum Cys-C (sCysC) or serum BMG (sBMG). However, it has been reported that $\mathrm{sCys-C}$ is increased by hyperthyroidism, human immunodeficiency virus (HIV) infection and steroids, while it is decreased by hypothyroidism, and taking cyclosporine [4-6]. In addition, it has been reported that sBMG is increased by inflammatory diseases, infections, malignant tumors, autoimmune diseases, and hyperthyroidism, while it is decreased by hypothyroidism [7, 8]. If eGFR is assumed to be inaccurate due to the above reasons, or each eGFR is significantly different, Cin, which provides an accurate GFR, will be required.

In clinical practice in Japan, inulin is used to evaluate kidney function in pediatric patients with chronic kidney disease (CKD), but in some cases it is used as a physiciandetermined method not as something having an approved dosage and administration. Therefore, the Japanese Society for Pediatric Nephrology examined the Cin measurement method, dosage and administration for Japanese pediatric patients, and developed a standard method with reference to the method proposed by Cole $[9,10]$. In the standard method, drinking water before and during the test, which is difficult for children, was changed to infusion with Ringer's solution. Furthermore, considering the difficulties associated with blood collection and the unreliability of urination in children, the number of blood and urine collections was reduced compared to the approved administration. In the standard method, the priming dose was calculated based on patient body weight, and the maintenance dose was calculated from individual eGFR and body surface area (BSA).

Members of the Japanese Society for Pediatric Nephrology conducted a clinical study to investigate any discrepancies between Cin measured by the standard method and 2-hour Ccr (2-h Ccr) measured at the same time [11]. They reported that the median 2-h Ccr and Cin in pediatric patients with renal disease was 121.6 and $74.0 \mathrm{~mL} /$ $\min / 1.73 \mathrm{~m}^{2}$, respectively, and found that 2 -h Ccr may overestimate Cin by approximately 1.5 -fold, regardless of the GFR value. In addition, on calculating the $\mathrm{Ccr} / \mathrm{Cin}$ value in pediatric patients with either CKD stage 2 or 3 using the study data, it was found that the lower limit (2.5th percentile value) of the $\mathrm{Ccr} / \mathrm{Cin}$ value in $95 \%$ of patients excluding the $2.5 \%$ above and below the Ccr/Cin value was 1.19 for stage 2 and 1.36 for stage 3 , respectively (data not shown). This result indicates that Ccr overestimates $\mathrm{Cin}$ by at least 1.2fold in pediatric patients with CKD stage 2 or 3 .

Generally, because pediatric nephrologists perform the Cin test using the standard method, in this study, we measured the Cin in pediatric patients (aged $<18$ years) with CKD stage 2 or 3 using a similar method. In addition, with reference to the previous study [11], we verified that $2-\mathrm{h} \mathrm{Ccr}$ overestimated $\mathrm{Cin}$ by at least 1.2 -fold, and confirmed the safety of inulin in pediatric patients.

\section{Materials and methods}

\section{Study design}

This was a phase 3 , open-label, single-dose study conducted at six institutions in Japan. Figure 1 shows the study schema. Patients who were judged to be eligible were hospitalized the day before inulin administration and discharged the following day.

\section{Inclusion and exclusion criteria}

Patients who met all inclusion criteria and did not fall under any exclusion criteria were enrolled in the study. Inclusion criteria for this study were as follows: patients with renal disease who require accurate GFR measurement (congenital anomalies of the kidney and urinary tract (CAKUT), reflux nephropathy, nephrotic syndrome, chronic glomerulonephritis, nephronophthisis, neurogenic bladder, polycystic kidney disease, Alport syndrome, etc.); Japanese patients aged 18 years or younger on the day of inulin administration; eGFR $30-89 \mathrm{~mL} / \mathrm{min} / 1.73 \mathrm{~m}^{2}$ calculated from the 
Fig. 1 Study schema. *Patients who had been treated with drugs that may affect serum inulin, sCre, sCys-C, and sBMG underwent a wash-out period

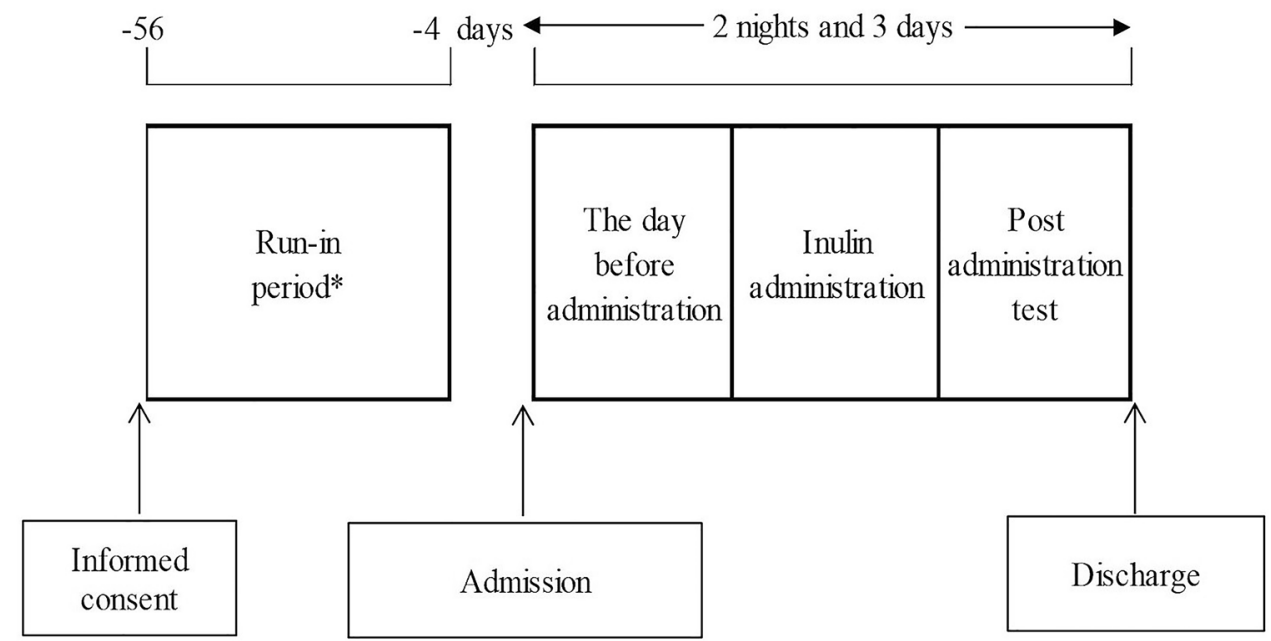

following Uemura's formula using sCre during the run-in period (sCys-C for patients aged under 2 years):

Males: eGFR $($ sCre $)=110.2 \times\left[\left(-1.259 x^{5}+7.815\right.\right.$ $\left.\left.x^{4}-18.57 x^{3}+21.39 x^{2}-11.71 x+2.628\right) / \mathrm{sCre}\right]+2.93[9]$

Females: eGFR $(\mathrm{sCre})=110.2 \times\left[\left(-4.536 x^{5}+27.16\right.\right.$ $\left.\left.x^{4}-63.47 x^{3}+72.43 x^{2}-40.06 x+8.778\right) / \mathrm{sCre}\right]+2.93$ [9]

Body length $(\mathrm{m})$ is expressed as " $x$ ".

eGFR (sCys-C) $=(104.1 /$ sCys-C) -7.80 [12]

Exclusion criteria for this study were as follows: patients who cannot urinate reliably by spontaneous urination, except for catheter urination; patients with edema (excluding those with mild acupressure impression), oliguria, and dehydration the day before inulin administration; patients with diseases that may affect sCre, sCys-C, and sBMG (neuromuscular disease, thyroid dysfunction, autoimmune disease, etc.); patients who used drugs that may affect serum inulin, sCre, sCys-C, and sBMG; patients with infections or inflammatory diseases (excluding chronic diseases) before inulin administration; patients with a history of allergy (including hypersensitivity) to inulin, food, drugs, and metals; patients with any other clinically significant medical conditions that could potentially preclude participation in this study.

\section{Efficacy endpoints}

The primary endpoint was the clearance ratio ( $\mathrm{Ccr} / \mathrm{Cin})$ between Cin and 2-h Ccr measured at the same time. The following secondary endpoints were set: 2 -h Ccr measured at the same time as Cin and eGFR calculated from sCre, sCys-C, and sBMG measured at the same time as Cin. The sCre, sCys-C and sBMG-based eGFR were calculated using the formulas shown below [9, 12-14]:

Males: eGFR $($ sCre $)=110.2 \times\left[\left(-1.259 x^{5}+7.815\right.\right.$ $\left.\left.x^{4}-18.57 x^{3}+21.39 x^{2}-11.71 x+2.628\right) / \mathrm{sCre}\right]+2.93$.

Females: eGFR $(\mathrm{sCre})=110.2 \times\left[\left(-4.536 x^{5}+27.16\right.\right.$ $\left.\left.x^{4}-63.47 x^{3}+72.43 x^{2}-40.06 x+8.778\right) / \mathrm{sCre}\right]+2.93$.
Body length ( $\mathrm{m})$ is expressed as " $x$ ".

If the patient was younger than 2 years, the above calculation result was multiplied by $\{0.107 \times \ln$ (Age $[$ month $])+0.656\}$.

eGFR $($ sCys-C) $=(104.1 / \mathrm{sCys}-\mathrm{C})-7.80$.

$\mathrm{eGFR}(\mathrm{sBMG})=(149.0 / \mathrm{sBMG})+9.15$.

According to the result of the previous study [11], the 2.5th percentile value of $\mathrm{Ccr} / \mathrm{Cin}$ in pediatric patients with CKD stage 2 was 1.19 . Therefore, the primary endpoint criterion for this study was set to 1.2 to confirm that the 2.5 th percentile value of Ccr/Cin for this study was higher than 1.19 .

\section{Safety evaluations}

In this study, adverse events (AEs) and safety assessments were conducted by clinical investigators based on vital signs, 12-lead electrocardiography, clinical laboratory tests, and clinical examinations. These were performed during run-in period, before and after inulin administration, and the next day, respectively. AEs were classified according to system organ class and preferred term (MedDRA version 22.1; Japanese Maintenance Organization, Tokyo, Japan) and were evaluated in terms of their potential causal relationship with inulin, as well as severity and seriousness. AEs judged to be related to the study drug were defined as adverse drug reactions (ADRs).

\section{Dosage and administration}

Figure 2 shows the dosage, administration, and method used to measure inulin. Patients fasted from at least 4 hours before the start of inulin administration. The inulin concentration was adjusted to $1.0 \mathrm{~g} / \mathrm{dL}$ in accordance with the package inserted. The priming rate was set at $8 \mathrm{~mL} / \mathrm{kg} / \mathrm{hr}$ (max: $300 \mathrm{~mL} / \mathrm{hr}$ ) for $30 \mathrm{~min}$. Subsequently, 
Fig. 2 Dosage and administration of inulin. *Calculated using sCre for patients aged 2 years or older and sCys-C for those younger than 2 years. $* *$ Calculated using the Haycock method. ***If urination was not observed, the administration duration could be extended to $150 \mathrm{~min}$

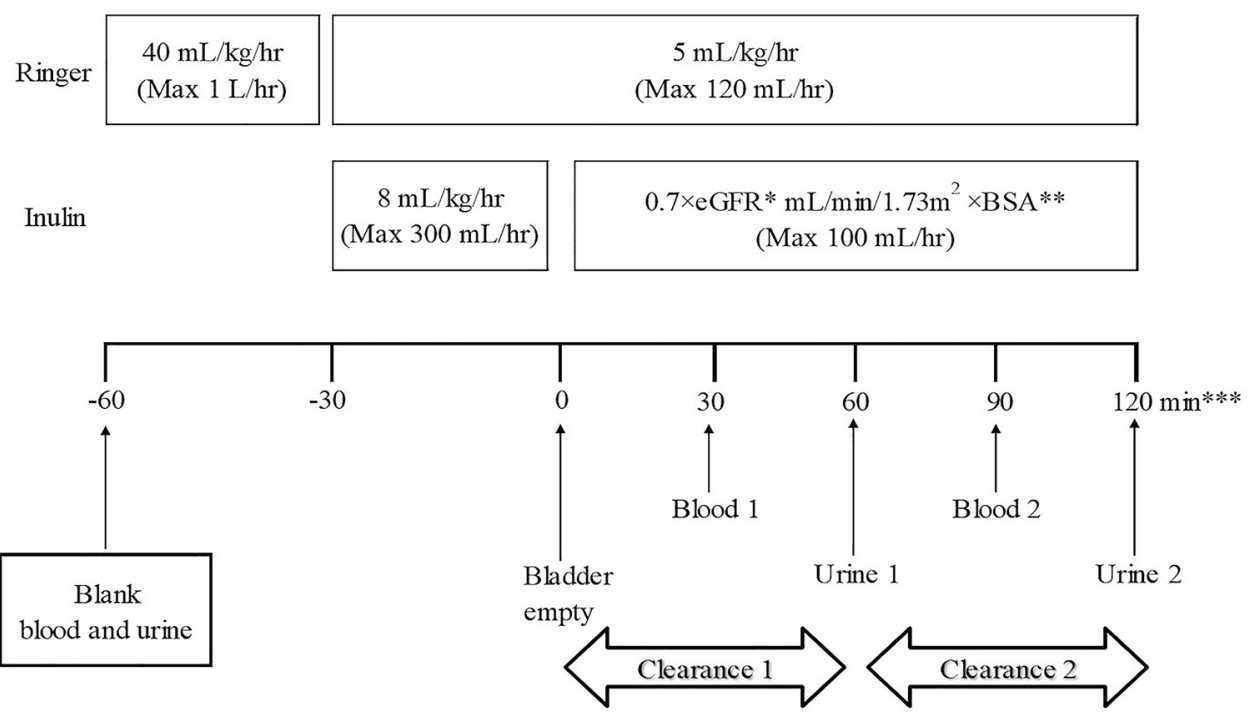

the maintenance rate was set at $0.7 \times \mathrm{eGFR} \mathrm{mL} /$ $\mathrm{min} / 1.73 \mathrm{~m}^{2} \times$ BSA (max: $100 \mathrm{~mL} / \mathrm{hr}$ ) for $120 \mathrm{~min}$ of continuous injection. This eGFR was calculated from the formula using sCre [9] for patients aged 2 years or older and the formula using sCys-C [12] for those younger than 2 years.

If no urine was passed during the first $120 \mathrm{~min}$, the administration period could be extended for up to $150 \mathrm{~min}$.

The dosage and administration of inulin in this study was set in accordance with the standard method formulated by the Japanese Society for Pediatric Nephrology. The target blood inulin concentration at this dosage is set to $20 \mathrm{mg} / \mathrm{dL}$ with reference to the method proposed by Cole [10]. The inulin dose required to reach the target blood concentration at the priming dose was calculated to be $40 \mathrm{mg} / \mathrm{kg}$, and the priming rate for $30 \mathrm{~min}$ was $8 \mathrm{~mL} /$ $\mathrm{kg} / \mathrm{hr}$. The maintenance dose was set to an administration rate obtained by the following formula: $0.7 \times \mathrm{eGFR}$ $\mathrm{mL} / \mathrm{min} / 1.73 \mathrm{~m}^{2} \times$ BSA taking into account the excretion of inulin in urine. BSA was calculated by the Haycock method [15]. Furthermore, instead of taking water by mouth, which is the usual practice in the approved method, Ringer's solution was intravenously infused at a rate of $40 \mathrm{~mL} / \mathrm{kg} / \mathrm{hr}(\max : 1 \mathrm{~L} / \mathrm{hr}$ ) from $30 \mathrm{~min}$ before inulin administration began, and thereafter continued at a rate of $5 \mathrm{~mL} / \mathrm{kg} / \mathrm{hr}$ (max: $120 \mathrm{~mL} / \mathrm{hr}$ ) until the end of inulin administration. Thirty minutes after the start of inulin administration, the bladder was emptied by urination, and the time at this point was set to 0 . Thereafter, blood samples were collected twice, first at $30 \mathrm{~min}$ and then at $90 \mathrm{~min}$. Urine samples were also obtained twice, first at $60 \mathrm{~min}$ and then at $120 \mathrm{~min}$.

\section{Statistical analyses}

The efficacy analysis was performed in the full analysis set (FAS), which comprised all patients who met the inclusion criteria and did not fall under the exclusion criteria, who had one or more efficacy endpoints measured, and who had no violations of Good Clinical Practice (GCP).

For the efficacy endpoint, we used the greater of the two calculated values for Cin, calculating the following summary statistics: $\mathrm{Ccr} / \mathrm{Cin}$ and the two-sided, $95 \%$ confidence intervals of the mean value. For the eGFR calculated using sCre, sCys-C, and sBMG and clearance ratio (eGFR/Cin), the two-sided $95 \%$ confidence intervals of the summary statistics and mean were similarly calculated.

The safety analysis was performed in the safety population (SP), which comprised all patients who received inulin, for whom safety information is available for evaluation, and who had no violations of GCP.

SAS software, version over 9.2 (SAS Institute, Cary, NC, USA) was used for statistical analysis. The study adopted a two-sided significance level of 5\% for all statistical analyses.

\section{Results}

\section{Patient flowcharts and baseline characteristics}

Figure 3 shows the flow diagram of the study protocol. Informed consent was obtained from 67 patients and 7 patients were withdrawn as they no longer met the inclusion criteria or came to fall under the exclusion criteria. Inulin was administered to the 60 patients confirmed to be eligible. Of these 60 patients, 1 patient was discontinued 
Fig. 3 Flow diagram of study protocol

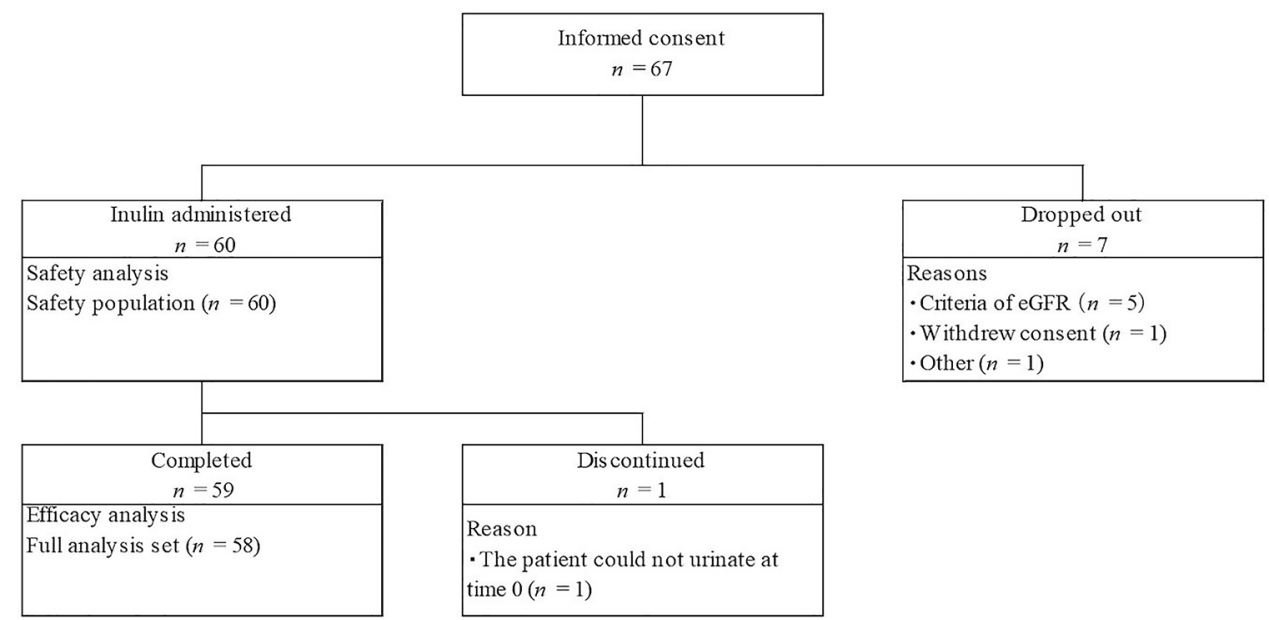

Table 1 Baseline characteristics of enrolled patients (FAS)

\begin{tabular}{lcc}
\hline Characteristic & $n(\%)$ & Mean \pm SD \\
\hline Sex & & \\
Male & $36(62.1)$ & \\
Female & $22(37.9)$ & \\
Age (year) & $1(1.7)$ & \\
$<2$ & $30(51.7)$ & \\
$\geq 2$ and $<12$ & $27(46.6)$ & \\
$\geq 12$ and $\leq 18$ & & \\
CKD stage & $33(56.9)$ & \\
2 & $17(29.3)$ & \\
3a & $8(13.8)$ \\
3b & & \\
Renal abnormality & \\
Congenital anomalies of the & $45(77.6)$ \\
$\quad$ kidney and urinary tract & \\
Reflux nephropathy & $4(6.9)$ \\
Nephrotic syndrome & $1(1.7)$ \\
Chronic glomerulonephritis & $2(3.4)$ \\
Neurogenic bladder & $4(6.9)$ \\
Alport's syndrome & $2(3.4)$ \\
Focal glomerulosclerosis & $2(3.4)$ \\
Hemolytic-uremic syndrome & $1(1.7)$ \\
Others & $2(3.4)$ \\
Height (cm) & & \\
Weight (kg) & & \\
eGFR (mL/min/1.73 m $\left.{ }^{2}\right)$ & \\
\hline
\end{tabular}

${ }^{a}$ If a subject fell into more than one category, they were counted more than once, so the total is greater than the number of cases

and 59 completed the study. In addition, 58 patients were included in the FAS, excluding 2 patients for whom efficacy data could not be obtained due to discontinuation or incomplete urine collection. The safety population (SP) comprised 60 patients who received inulin. Table 1 shows
Table 2 Result of Ccr, Cin and Ccr/Cin efficacy endpoints

\begin{tabular}{lllll}
\hline Parameters & $n$ & Mean & SD & $\begin{array}{l}95 \% \text { confidence } \\
\text { interval }\end{array}$ \\
\hline Ccr & 58 & 99.1 & 30.1 & $91.2-107.0$ \\
Cin & 58 & 57.9 & 19.8 & $52.7-63.1$ \\
Ccr/Cin & 58 & 1.78 & 0.52 & $1.6406-1.9142$ \\
One-sample $t$ test $^{\mathrm{a}}{ }^{\mathrm{a}}$ & $p$ value & & & $p<0.001$ \\
& $95 \%$ confidence interval & $1.6406-1.9142$ \\
\hline
\end{tabular}

${ }^{a}$ The null hypothesis was " $\mathrm{Ccr} / \mathrm{Cin}=1.2$ "

the baseline characteristics of the patients (FAS) included in this study. There were 36 males (62.1\%) and 22 females (37.9\%). The height, weight and eGFR (mean \pm SD) were $134.71 \pm 25.51 \mathrm{~cm}, 34.36 \pm 16.30 \mathrm{~kg}$, and $63.4 \pm 15.6$ $\mathrm{mL} / \mathrm{min} / 1.73 \mathrm{~m}^{2}$, respectively. There was 1 patient aged 1 year (1.7\%), 30 patients aged from 2 to 11 years (51.7\%), and 27 patients aged from 12 to 18 years (46.6\%). The most common causative disease was CAKUT in 45 patients $(77.6 \%)$.

\section{Efficacy}

The Cin and Ccr (mean $\pm \mathrm{SD}$ ), measured at the same time, were $57.9 \pm 19.8$ and $99.1 \pm 30.1 \mathrm{~mL} / \mathrm{min} / 1.73 \mathrm{~m}^{2}$, respectively. The clearance ratio (Ccr/Cin) between $\mathrm{Cin}$ and $\mathrm{Ccr}$ was $1.78 \pm 0.52($ mean $\pm \mathrm{SD})$. In addition, the lower limit of the two-sided $95 \%$ confidence interval of the mean value was 1.6406, exceeding the preset criterion of $1.2(p<0.001$, 1 -sample $t$ test) (Table 2). Figure 4 shows the scatter plot of $\mathrm{Ccr}$ and Cin. This result showed that the Ccr exceeded the Cin in all patients.

The eGFR (mean $\pm \mathrm{SD}$ ) calculated from sCre, sCys$\mathrm{C}$, and sBMG measured at the same time as Cin was $68.2 \pm 17.7,79.5 \pm 24.3$, and $78.5 \pm 23.1 \mathrm{~mL} / \mathrm{min} / 1.73 \mathrm{~m}^{2}$, 


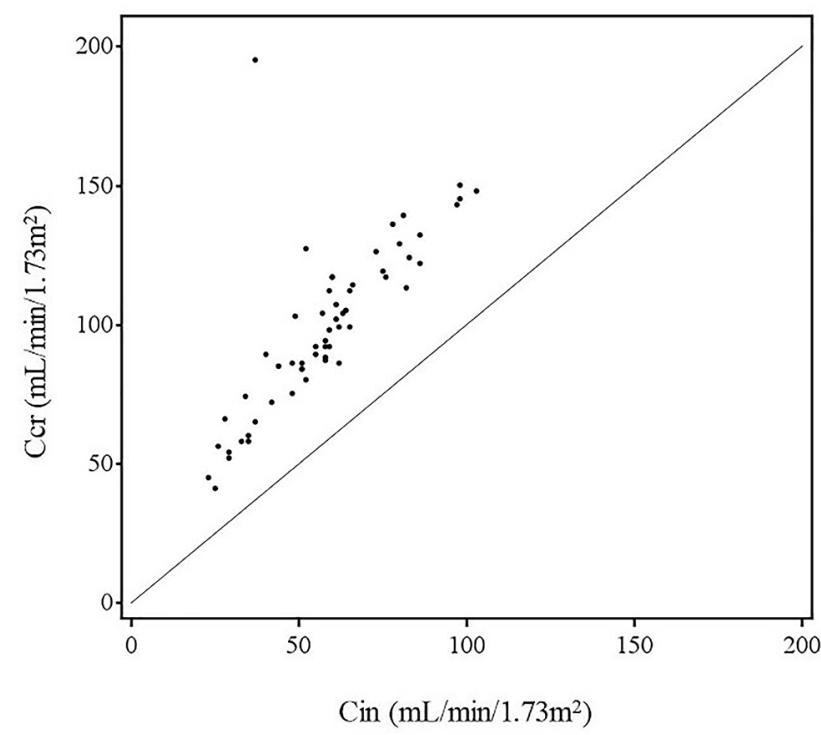

Fig. 4 Scatter plot of Ccr and Cin

Table 3 Result of eGFR and relationship with Cin

\begin{tabular}{llcccc}
\hline Parameters & $\begin{array}{l}\text { Parameters used } \\
\text { to calculate eGFR }\end{array}$ & $n$ & Mean & SD & $\begin{array}{l}\text { 95\% confidence } \\
\text { interval }\end{array}$ \\
\hline eGFR & Cre & 58 & 68.2 & 17.7 & $63.5-72.9$ \\
& Cys-C & 58 & 79.5 & 24.3 & $73.2-85.9$ \\
& BMG & 58 & 78.5 & 23.1 & $72.4-84.6$ \\
eGFR/Cin & Cre & 58 & 1.24 & 0.28 & $1.1642-1.3103$ \\
& Cys-C & 58 & 1.42 & 0.35 & $1.3313-1.5170$ \\
& BMG & 58 & 1.40 & 0.28 & $1.3292-1.4779$ \\
\hline
\end{tabular}

Table 4 Incidences of adverse events

\begin{tabular}{llll}
\hline Adverse events & \multicolumn{3}{l}{ Safety population $(n=60)$} \\
\cline { 2 - 4 } & $\begin{array}{l}\text { Number of } \\
\text { events }\end{array}$ & $\begin{array}{l}\text { Number of } \\
\text { patients }\end{array}$ & Incidence (\%) \\
\hline All & 5 & 4 & 6.7 \\
Conjunctivitis & 1 & 1 & 1.7 \\
Vomiting & 1 & 1 & 1.7 \\
Injection site pain & 1 & 1 & 1.7 \\
Hypoglycaemia & 1 & 1 & 1.7 \\
Presyncope & 1 & 1 & 1.7 \\
\hline
\end{tabular}

The adverse event name is shown by preferred term per MedDRA version 22.1

respectively. The clearance ratios (eGFR/Cin) were $1.24 \pm 0.28,1.42 \pm 0.35$, and $1.40 \pm 0.28($ mean $\pm S D)$, respectively (Table 3 ).

\section{Safety}

Table 4 shows all AEs that occurred during the study. The incidence of AEs was 6.7\% (four patients), and no serious adverse events were observed. Vomiting and hypoglycemia occurred in one patient were moderate in severity. These two events may have occurred because the patient continued to fast, thus a causal relationship with inulin was ruled out.

Neither severe AEs nor AEs leading to discontinuation of the study were observed. No ADRs were reported.

Accordingly, no major safety issues were observed in any of the pediatric patients with renal disease.

\section{Discussion}

The approved dosage and administration of inulin is set to a uniform dose regardless of body type or renal function. In children, because there is a large difference in body size depending on age, if they were to be administered the same dose as adults, this could lead to an overdose. Therefore, dosage according to the standard method is calculated based on individual body type and level of renal function. In addition, in the approved method, water has to be drunk before and during measurement to ensure that a sufficient amount of urine is generated, but drinking a large amount of water may be difficult for children. Accordingly, infusion of Ringer's solution is adopted instead of drinking water. The infusion rate of Ringer's solution is calculated based on body weight and the infusion is continued until the end of inulin administration. Furthermore, considering that the number of blood and urine collections and the intervals between collections in the approved method are also troublesome for children, in the standard method, there are two sample collections at 60-min intervals for both blood and urine.

In this study, Cin in pediatric patients with CKD stage 2 or 3 was measured using a method based on the standard method. The $\mathrm{Cin}$ and $\mathrm{Ccr}($ mean $\pm \mathrm{SD})$ measured at the same time were $57.9 \pm 19.8$ and $99.1 \pm 30.1 \mathrm{~mL} / \mathrm{min} / 1.73 \mathrm{~m}^{2}$, respectively. The Ccr/Cin was $1.78 \pm 0.52($ mean $\pm \mathrm{SD})$, which was confirmed to exceed 1.2. In addition, we compared the results of this study to those obtained in previous study. The previous study showed that the median values of Cin and Ccr were 74.0 and $121.6 \mathrm{~mL} / \mathrm{min} / 1.73 \mathrm{~m}^{2}$, respectively, and $\mathrm{Ccr} / \mathrm{Cin}$ was about 1.6. Furthermore, they reported that $\mathrm{Ccr} / \mathrm{Cin}$ increases as renal function declines [11]. In this study, the median values of Cin and Ccr were 58.0 and $98.5 \mathrm{~mL} / \mathrm{min} / 1.73 \mathrm{~m}^{2}$, respectively, and the median $\mathrm{Ccr} / \mathrm{Cin}$ was 1.67. The median $\mathrm{Ccr} / \mathrm{C}$ in was calculated to be $1.65,1.67$, and 1.78 for CKD stage 2, 3a, and 3b, respectively, suggesting that $\mathrm{Ccr} / \mathrm{Cin}$ tended to increase as renal function declined. It has been reported that $\mathrm{Ccr} / \mathrm{Cin}$ generally tends to increase as renal function declines [16]. Since 
the results of this study and previous study confirmed this tendency, Cin using the standard method was considered to accurately reflect kidney function.

For patients whose sCre level may not be an accurate reflection of renal function, eGFR using sCys-C or sBMG may be useful $[12,13]$. In this study, eGFR (mean \pm SD) calculated from sCre, sCys-C, and sBMG measured at the same time as Cin was $68.2 \pm 17.7,79.5 \pm 24.3$, and $78.5 \pm 23.1 \mathrm{~mL} / \mathrm{min} / 1.73 \mathrm{~m}^{2}$, respectively, and eGFR/Cin $($ mean $\pm S D)$ was $1.24 \pm 0.28,1.42 \pm 0.35$, and $1.40 \pm 0.28$, respectively. It was revealed that the eGFR calculated from sCre was the closest to Cin, and the eGFR values calculated from sCys-C and sBMG were similar. These results suggest that although all eGFR values are closer to Cin than Ccr, each eGFR may overestimate renal function compared to Cin. Therefore, we believe that Cin measurement using this standard method is necessary to ensure more accurate evaluation of renal function in pediatric patients with renal disease.

Cin may show a slightly lower value than the original kidney function if the urine volume is not measured accurately due to insufficient urine storage. In this study, we used the greater of the two calculated values for Cin, as does the standard method due to concerns about insufficient urine collection. However, it is unclear whether sufficient urine could be collected from pediatric patients, particularly from infants. Therefore, the possibility cannot be ruled out that eGFR/Cin tended to be slightly higher than in the original study and some differences may be observed because eGFR is not affected by urine volume. Previous study observed slight differences between eGFR and Cin [17]. Additionally, although the pediatric GFR estimation formula was created by measuring Cin in patients with CKD stage 1 to 5, many patients with mild renal dysfunction were included. On the other hand, this study only included patients with CKD stage 2 and 3. This difference in patient background may have caused discrepancy in Cin and eGFR. To verify how much the discrepancy between Cin and the eGFR from formula, a comparative study with many patients is required.

Regarding the safety of this study, none of the patients developed edema due to the infusion of Ringer's solution. No ADRs, serious adverse events, or discontinuation due to adverse events were observed. In short, there were no safety issues.

In summary, the method used to measure Cin in this study provides clinicians with a simpler measurement method for pediatric renal disease patients than the approved method, making it easier to evaluate renal function accurately through the use of Cin. For pediatric patients with renal disease, if evaluation of renal function by Ccr or eGFR is not an option, evaluation of renal function using the Cin measurement method proposed in this study will make it possible to determine treatment strategies such as appropriate drug selection and management of complications.

\section{Limitations}

This study was conducted on a small number of cases with just one patient under 2 years of age. In addition, patients with CKD stage 2 and 3 were targeted, and most of the renal abnormalities were CAKUT.

Acknowledgements We are grateful to investigators, local study coordinators, and the patients for their valuable contributions to this study.

\section{Declarations}

Conflict of interest OU has received consultant fees from Fuji Yakuhin Co., Ltd., the manufacturer of inulin. The other authors have declared that no conflict of interest exists.

Ethical approval The study was conducted according to Good Clinical Practice guidelines, the Declaration of Helsinki and other applicable regulatory requirements. The institutional review board at each institution approved the conduct of the study and clinical study protocol, informed consent form, investigator's brochures, and other documents. The study information has been registered with the U.S National Library of Medicine (ClinicalTrials.gov Identifier: NCT03345316).

Informed consent Informed consent was obtained from all individual participants included in the study.

Open Access This article is licensed under a Creative Commons Attribution 4.0 International License, which permits use, sharing, adaptation, distribution and reproduction in any medium or format, as long as you give appropriate credit to the original author(s) and the source, provide a link to the Creative Commons licence, and indicate if changes were made. The images or other third party material in this article are included in the article's Creative Commons licence, unless indicated otherwise in a credit line to the material. If material is not included in the article's Creative Commons licence and your intended use is not permitted by statutory regulation or exceeds the permitted use, you will need to obtain permission directly from the copyright holder. To view a copy of this licence, visit http://creativecommons.org/licenses/by/4.0/.

\section{References}

1. Smith HW. Measurement of the rate of glomerular filtration. Oxford University Press; 1956. p. 25-35.

2. Shemesh O, Golbetz H, Kriss JP, Myers BD. Limitations of creatinine as a filtration marker in glomerulopathic patients. Kidney Int. 1985;28:830-8.

3. Osamu U, Masataka H, Takeshi M, et al. Age, gender, and body length effects on reference serum creatinine levels determined by an enzymatic method in Japanese children: a multicenter study. Clin Exp Nephrol. 2011;15:694-9.

4. Gordana D, Danica S, Khawla AM, et al. Higher levels of cystatin $\mathrm{C}$ in HIV/AIDS patients with metabolic syndrome. Basic Clin Pharmacol Toxicol. 2018;122:396-401. 
5. Kotajima N, Yanagawa Y, Aoki T, et al. Influence of thyroid hormones and transforming growth factor- $\beta 1$ on cystatin $\mathrm{C}$ concentrations. J Int Med Res. 2010;38:1365-73.

6. Uemura $\mathrm{O}$, Iwata $\mathrm{N}$, Nagai $\mathrm{T}$, et al. Influence of thyroid function on glomerular filtration rate and other estimates of kidney function in two pediatric patients. CEN Case Rep. 2018;7:169-73.

7. Bülent Y, Seyfettin K, Osman Y, Serap A. Serum beta 2-microglobulin as a biomarker in inflammatory bowel disease. World J Gastroenterol. 2014;20:10916-20.

8. Roiter I, Da Rin G, De Menis E, et al. Increased serum beta 2-microglobulin concentrations in hyperthyroid states. J Clin Pathol. 1991;44:73-4.

9. Uemura O, Nagai T, Ishikura K, et al. Creatinine-based equation to estimate the glomerular filtration rate in Japanese children and adolescents with chronic kidney disease. Clin Exp Nephrol. 2014;18:626-33.

10. Cole BR, Giangiacomo J, Ingelfinger JR, Robson AM. Measurement of renal function without urine collection. A critical evaluation of the constant-infusion technic for determination of inulin and para-aminohippurate. N Engl J Med. 1972;287:1109-14.

11. Uemura O, Nagai T, Yamakawa S, et al. Assessment of kidney function in children by enzymatic determination of 2- or 24-h creatinine clearance: comparison with inulin clearance. Clin Exp Nephrol. 2016;20:462-8.
12. Uemura $\mathrm{O}$, Nagai $\mathrm{T}$, Ishikura $\mathrm{K}$, et al. Cystatin C-based equation to estimating the glomerular filtration rate in Japanese children and adolescents. Clin Exp Nephrol. 2014;18:718-25.

13. Ikezumi $Y$, Uemura $\mathrm{O}$, Nagai $\mathrm{T}$, et al. Beta-2 microglobulin-based equation for estimating glomerular filtration rates in Japanese children and adolescents. Clin Exp Nephrol. 2015;19:450-7.

14. Uemura $\mathrm{O}$, Ishikura $\mathrm{K}$, Gotoh $\mathrm{Y}$, et al. Creatinine-based estimated glomerular filtration rate for children younger than 2 years. Clin Exp Nephrol. 2018;22:483-4.

15. Haycock GB, Schwartz GJ, Wisotsky DH. Geometric method for measuring body surface area: a height-weight formula validated in infants, children, and adults. J Pediatr. 1978;93:62-6.

16. Levey AS. Measurement of renal function in chronic renal disease. Kidney Int. 1990;38:167-84.

17. Gotoh Y, Uemura O, Ishikura K, et al. Validation of estimated glomerular filtration rate equations for Japanese children. Clin Exp Nephrol. 2018;22:931-7.

Publisher's Note Springer Nature remains neutral with regard to jurisdictional claims in published maps and institutional affiliations. 